\title{
Investigations on the Hyers-Ulam stability of generalized radical functional equations
}

\author{
JAnusz BrZdęK(D, El-SAYEd El-HAdy, AND JENs Schwaiger \\ Dedicated to Professor János Aczél on the occasion of his 95th birthday.
}

\begin{abstract}
In (Brzdęk and Schwaiger in Aeq Math 92: 975-991, 2018) solutions of far reaching generalizations of the so-called radical functional equation $f(p(\pi(x)+\pi(y)))=f(x)+f(y)$ have been investigated. These investigations are continued here by analysing the corresponding stability results, which have been the main subject of several recent papers. We propose a very general and uniform approach.
\end{abstract}

Mathematics Subject Classification. 39B82.

Keywords. Cauchy equation, Groupoid, Hyers-Ulam stability, Radical functional equation, Square symmetric operation.

\section{Introduction and preliminaries}

The main subject of this paper is stability of the so-called generalized radical functional equation (23), which is a natural generalization of the functional equation

$$
f\left(\sqrt{x^{2}+y^{2}}\right)=f(x)+f(y),
$$

considered for functions with the set of reals $\mathbb{R}$ as the domain (see, e.g., [21]). For more information on the equations and examples of recent results we refer to $[5,7,12,20]$.

We present a kind of very general approach to that subject. To this aim we use some nonstandard ways of measuring a distance in a set. So we start the paper with several definitions, results and simple observations on that subject.

Various ways of measuring distance are useful in applied sciences (see, e.g., [11]). But there is some confusion in the terminology concerning them; in particular, various generalizations of the notion of metric. For example, according 
to [28], we have the following definition of a quasi-metric space $\left(\mathbb{R}_{+}\right.$stands for the set of nonnegative reals).

Definition 1. Let $X$ be a set with at least 2 elements. Then $d: X \times X \rightarrow \mathbb{R}_{+}$ is called quasi-metric if there is some $K^{\prime} \in \mathbb{R}$ such that

(a) $d(x, y)=0$ if and only if $x=y$

(b) $d(x, y)=d(y, x)$ for all $x, y \in X$ and

(c) $d(x, y) \leq K^{\prime} \max (d(x, z), d(z, y))$ for all $x, y, z \in X$.

However, in several other papers (see, e.g., [11]), a quasi-metric is a function $d: X \times X \rightarrow \mathbb{R}_{+}$satisfying only condition (a) and the usual triangle inequality:

$$
d(x, y) \leq d(x, z)+d(z, y) \text { for all } x, y, z \in X .
$$

There are few other ambiguities in this area.

Remark 1. Note that we must have $K^{\prime} \geq 1$, in Definition 1 , in view of the properties (a) and (c) with $x \neq y=z$.

In this paper we also measure a distance in a nonempty set $X$ using functions $d: X \times X \rightarrow \mathbb{R}_{+}$satisfying only the following two conditions

(I) if $d(x, y)=d(y, x)=0$, then $x=y$;

(II) $d(x, y) \leq K(d(x, z)+d(z, y))$,

for all $x, y, z \in X$ and with some $K \in \mathbb{R}_{+}$. If $K=1$, then such a function is often called a dislocated quasi-metric (see, e.g., [25,27]), or shortly: dqmetric. The notion of a dq-metric space is a natural generalization of the usual definitions of metric, quasimetric, partial metric and metric-like spaces and plays crucial roles in computer science and cryptography (see, e.g., $[2,8$, $11,19,25,27])$.

We will use that type of terminology and to be short and precise, given an arbitrary $K \in \mathbb{R}_{+}$, by dq- $K$-metric we understand every function $d: X \times X \rightarrow$ $\mathbb{R}_{+}$fulfilling conditions (I) and (II).

Remark 2. Actually conditions (c) and (II) are closely related as we may see below.

1. Property (c) implies (II) with $K=2 K^{\prime}$.

2. (II) implies property (c) with $K^{\prime}=K$.

Remark 3. Clearly, if $K \in(1, \infty)$, then every function $d: \mathbb{R}^{2} \rightarrow\{0\} \cup[1 / K, 1]$ with $d^{-1}(\{0\}) \subset\{(x, x): x \in \mathbb{R}\}$ is a dq- $K$-metric.

Further, let $p \in(1, \infty)$ and $(Z, d)$ be a dq-metric space. Then it is easy to show that $d_{p}$ is a dq- $2^{p-1}$-metric, where

$$
d_{p}(x, y):=d(x, y)^{p}, \quad x, y \in Z .
$$

In fact, for every $x, y, z \in Z$, we have

$$
\begin{aligned}
d_{p}(x, y) & \leq(d(x, z)+d(z, y))^{p} \leq 2^{p-1}\left(d(x, z)^{p}+d(z, y)^{p}\right) \\
& =2^{p-1}\left(d_{p}(x, z)+d_{p}(z, y)\right),
\end{aligned}
$$


because $(a+b)^{p} \leq 2^{p-1}\left(a^{p}+b^{p}\right)$ for $a, b \in[0, \infty)$.

Remark 4. Let $a, b \in(0, \infty), n, k \in \mathbb{N}$ (positive integers), and $\alpha: \mathbb{R} \rightarrow \mathbb{R}_{+}$ be such that $\alpha^{-1}(\{0\})=\{0\}$. Then it is easy to check that the function $d: \mathbb{R} \times \mathbb{R} \rightarrow \mathbb{R}_{+}$, given by any of the following six simple formulas, is a dqmetric:

$$
\begin{aligned}
& d(x, y)=\alpha(x), \quad x, y \in \mathbb{R}, \\
& d(x, y)=\max \left\{a|x|^{k}, b|y|^{n}\right\}, \quad x, y \in \mathbb{R}, \\
& d(x, y)=a|x|^{k}+b|y|^{n}, \quad x, y \in \mathbb{R}, \\
& d(x, y)=\sqrt{a|x|^{k}+b|y|^{n}}, \quad x, y \in \mathbb{R}, \\
& d(x, y)=\sqrt[n]{\max \{x-y, 0\}}, \quad x, y \in \mathbb{R}, \\
& d(x, y)=\max \{x-\lfloor y\rfloor, 0\}, \quad x, y \in \mathbb{R},
\end{aligned}
$$

where $\lfloor\cdot\rfloor$ is the floor function, i.e., $\lfloor y\rfloor:=\max \{n \in \mathbb{Z}: n \leq y\}$ ( $\mathbb{Z}$ stands for the set of integers).

Next, if $n \in \mathbb{N}, a_{1}, \ldots, a_{n} \in[0, \infty)$ and $d_{1}, \ldots, d_{n}$ are dq-metrics in a nonempty set $X$, then $d$ and $d_{0}$ also are dq-metrics in $X$, where

$$
d(x, y)=\sum_{i=1}^{n} a_{i} d_{i}(x, y), \quad d_{0}(x, y)=\max _{i=1, \ldots, n} a_{i} d_{i}(x, y), \quad x, y \in X .
$$

For some further examples we refer to, e.g., $[2,8,19]$ and the references therein. However, the next remark provides possible generalizations of the examples of dq-metrics presented above.

Remark 5. The following very general example of a dq-metric, on a nonempty set $X$, covers the first four examples in the previous remark.

Namely, let $x_{0} \in X$ and $\varphi, \psi: X \rightarrow \mathbb{R}_{+}, \nu: \mathbb{R}_{+}^{2} \rightarrow \mathbb{R}_{+}$satisfy

$$
\begin{aligned}
\varphi^{-1}(\{0\})= & \left\{x_{0}\right\}, \quad \nu^{-1}(\{0\}) \subset\left\{(0, x): x \in \mathbb{R}_{+}\right\}, \\
& \max \{\nu(u, 0), \nu(0, v)\} \leq \nu(u, v) \leq \nu(u, 0)+\nu(0, v),(u, v) \in \mathbb{R}_{+}^{2} .
\end{aligned}
$$

Then it is easy to verify that $d: X^{2} \rightarrow \mathbb{R}_{+}$, defined by $d(x, y):=\nu(\varphi(x), \psi(y))$, is a dq-metric, because for each $t, u, v, w \in \mathbb{R}_{+}$,

$$
\nu(u, v) \leq \nu(u, 0)+\nu(0, v) \leq \nu(u, w)+\nu(t, v) .
$$

Next, if $d$ is a dq-metric, then so is $d_{p}, d_{p}(x, y):=d(x, y)^{p}$, for all $0<p \leq 1$, since $(a+b)^{p} \leq a^{p}+b^{p}$ for $a, b \in \mathbb{R}_{+}$. This generalizes the 4 th and 5 th example.

A generalization of the 6th example is given by $d(x, y):=\max \{\varphi(x)-$ $\psi(y), 0\}$, where $X$ is a nonempty set and $\varphi, \psi: X \rightarrow \mathbb{R}$ satisfy the following two conditions.

1. $\varphi$ is injective and $\psi \leq \varphi$.

2. For all $x, y$ the inequality $\varphi(x) \leq \varphi(y)$ implies $\psi(x) \leq \psi(y)$. 
In fact, let $d(x, y)=d(y, x)=0$. Then $\varphi(x) \leq \psi(y)$ and $\varphi(y) \leq \psi(x)$. Thus $\varphi(x) \leq \psi(y) \leq \varphi(y) \leq \psi(x) \leq \varphi(x)$ and therefore $\varphi(x)=\varphi(y)$ implying $x=y$.

Further, if $\varphi(x)-\psi(y) \leq 0$, then the triangle inequality trivially holds.

So, let $\varphi(x)-\psi(y)>0$. First assume that $\varphi(x)-\psi(z) \leq 0$. Then $\varphi(z)-$ $\psi(y)>0$, because otherwise $\varphi(z) \leq \psi(y)$ implying $\varphi(x) \leq \psi(z) \leq \varphi(z) \leq \psi(y)$ and thus contradicting $\varphi(x)>\psi(y)$. So $\varphi(x)-\psi(z) \leq 0, \varphi(z)-\psi(y)>0$, which implies that $\varphi(x) \leq \psi(z) \leq \varphi(z)$ and

$$
d(x, y)=\varphi(x)-\psi(y) \leq \varphi(z)-\psi(y)=d(z, y) \leq d(x, z)+d(z, y) .
$$

Finally, let $\varphi(x)-\psi(z)>0$. Consider two cases.

Case 2.1) $\varphi(z)-\psi(y) \leq 0$. Then $\psi(z) \leq \varphi(z) \leq \psi(y)$ and consequently we have $\varphi(x)-\psi(y) \leq \varphi(x)-\psi(z)$, whence $d(x, y) \leq d(x, z) \leq d(x, z)+d(z, y)$.

Case 2.2) $\varphi(z)-\psi(y)>0$. Then $d(x, z)+d(z, y)=\varphi(x)-\psi(z)+\varphi(z)-$ $\psi(y)=\varphi(x)-\psi(y)+(\varphi(z)-\psi(z))=d(x, y)+(\varphi(z)-\psi(z)) \geq d(x, y)$.

Let us mention yet, that a function $d: X^{2} \rightarrow \mathbb{R}_{+}$such that (a), (b) and (II) are valid, have been called a $b$-metric (cf., e.g., [13]). That term has been used for the first time in [9] with $K=2$ and next in [10] for any $K \geq 1$. In what follows we say that $(X, d, K)$ is a b-metric space provided $d: X^{2} \rightarrow \mathbb{R}_{+}$ is a b-metric with (II) fulfilled.

The following two examples have been provided in [4, Examples 1.1 and $1.2]$.

Example 1. Let $X$ be a Banach space, $p \in(0,1)$,

$$
\ell_{p}(X):=\left\{\left(x_{n}\right)_{n \in \mathbb{N}} \in X^{\mathbb{N}}: \sum_{n=1}^{\infty}\left\|x_{n}\right\|^{p}<\infty\right\},
$$

and $\rho: \ell_{p}(X)^{2} \rightarrow[0, \infty)$ be defined by

$$
\rho(x, y):=\left(\sum_{n=1}^{\infty}\left\|x_{n}-y_{n}\right\|^{p}\right)^{1 / p}, \quad x=\left(x_{n}\right)_{n \in \mathbb{N}}, y=\left(y_{n}\right)_{n \in \mathbb{N}} \in \ell_{p}(X) .
$$

Then $\left(\ell_{p}(X), \rho, 2^{1 / p}\right)$ is a $b$-metric space.

Example 2. If $p \in(0,1)$,

$$
L_{p}[0,1]:=\left\{x:[0,1] \rightarrow \mathbb{R}: \int_{0}^{1}|x(t)|^{p} d t<1\right\},
$$

and $\rho: L_{p}[0,1] \times L_{p}[0,1] \rightarrow \mathbb{R}_{+}$is defined by

$$
\rho(x, y):=\left(\int_{0}^{1}|x(t)-y(t)|^{p} d t\right)^{1 / p}, \quad x, y \in L_{p}[0,1],
$$

then $\left(L_{p}[0,1], \rho, 2^{1 / p}\right)$ is a $b$-metric space.

Let us yet recall the following very interesting result. 
Theorem 1. [24, Proposition, p. 4308] Let $(Y, d, \eta)$ be a $b$-metric space and $D_{d}(x, y)=\inf \left\{\sum_{i=1}^{n} d^{\xi}\left(x_{i}, x_{i+1}\right): x_{2}, \ldots, x_{n} \in Y, n \in \mathbb{N}, x_{1}=x, x_{n+1}=y\right\}$

for all $x, y \in Y$, where $\xi:=\log _{2 \eta} 2$ and $d^{\xi}(x, y)=(d(x, y))^{\xi}$ for $x, y \in Y$. Then $D_{d}$ is a metric in $Y$ with

$$
\frac{1}{4} d^{\xi}(x, y) \leq D_{d}(x, y) \leq d^{\xi}(x, y), \quad x, y \in Y
$$

In particular, if $d$ is a metric, then $D_{d}=d$.

Remark 6. Assume that $(X,\|\cdot\|, \eta)$ is a real quasi-normed space, i.e., $\eta \geq 1$ is a fixed real number, $X$ is a real (complex, respectively) linear space and $\|\cdot\|: X \rightarrow \mathbb{R}_{+}$satisfy the following three conditions:

(a1) $\|x\|=0$ if and only if $x=0$;

(b1) $\|\alpha x\|=|\alpha|\|x\|$ for $x \in X$ and $\alpha \in \mathbb{R}(\alpha \in \mathbb{C}$, resp.);

(c1) $\|x+y\| \leq \eta(\|x\|+\|y\|)$ for $x, y \in X$.

Let $Y \subset X$ be nonempty and $d: Y^{2} \rightarrow \mathbb{R}_{+}$be given by: $d(x, y):=\|x-y\|$ for $x, y \in Y$. Then it is easily seen that $(Y, d, \eta)$ is a b-metric space with $d(\alpha x, \alpha y)=|\alpha| d(x, y)$ for $x, y \in X$ and $\alpha \in \mathbb{R}(\alpha \in \mathbb{C}$, resp. $)$. Note yet that such $d$ is also invariant (i.e., $d(x+z, y+z)=d(x, y)$ for $x, y, z \in Y)$.

In view of Aoki-Rolewicz Theorem (see, e.g., [22, Theorem 1]; cf. also [23]), each quasi-norm is equivalent to some $p$-norm. Let us remind here that, if $X$ is a a real (complex, respectively) linear space and $p>0$, then a mapping $\|\cdot\|: X \rightarrow \mathbb{R}_{+}$is a $p$-norm provided conditions (a1) and (b1) hold and

(c2) $\|x+y\|^{p} \leq\|x\|^{p}+\|y\|^{p}$ for $x, y \in X$.

Let $d$ be a dq-K-metric in a nonempty set $Y$. We say that $x \in Y$ is a limit of a sequence $\left(x_{n}\right)_{n=1}^{\infty}$ in $Y$ provided

$$
\lim _{n \rightarrow \infty} \max \left\{d\left(x_{n}, x\right), d\left(x, x_{n}\right)\right\}=0 ;
$$

then we write $x_{n} \rightarrow x$ or $x=\lim _{n \rightarrow \infty} x_{n}$; in view of (I), it is easy to note that such a limit must be unique, because

$$
d(x, y) \leq d\left(x, x_{n}\right)+d\left(x_{n}, y\right), \quad d(y, x) \leq d\left(y, x_{n}\right)+d\left(x_{n}, x\right)
$$

for every $y \in Y$ and $n \in \mathbb{N}$.

Next, we say that a sequence $\left(x_{n}\right)_{n=1}^{\infty}$ in $Y$ is Cauchy if

$$
\lim _{N \rightarrow \infty} \sup _{m, n \geqslant N} d\left(x_{n}, x_{m}\right)=0 ;
$$

$d$ is complete if every Cauchy sequence in $Y$ has a limit in $Y$.

Remark 7. Usually, in a dq-metric space, the Cauchy sequence can be defined in a somewhat different way; e.g., in a metric-like space $(Y, d)$, a sequence

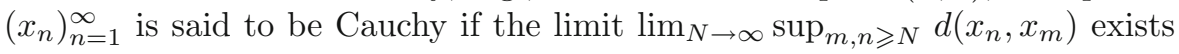


and is finite (see [3]). But such definitions are too weak and would exclude from our considerations the metric and quasi-metric spaces. The same concerns the notion of completeness.

Also our definition of a limit of a sequence is stronger than the usual (in a dq-metric space), but this seems to be necessary in the proof of the main result; moreover, it actually corresponds to our definition of the Cauchy sequence and makes such limit unique (which is not the case in general) and therefore more useful.

In the framework of [26] the idea of a quasi- $\beta$-normed space has been used. This paper contains further references on this topic. To obtain an even more general setting we use the following modification of the concept.

Definition 2. Let $X$ be a vector space over $\mathbb{K} \in\{\mathbb{R}, \mathbb{C}\}, K \geq 1, \beta \geq 0$. Then \|\|$: X \rightarrow \mathbb{R}$ is called quasi- $\beta$-norm if

1. $\|x\| \geq 0$ for all $x \in X$ and $\|x\|=0$ if and only if $x=0$

2. $\|\lambda x\|=|\lambda|^{\beta}\|X\|$ for all $x \in X, \lambda \in \mathbb{K}$.

3. $\|x+y\| \leq K(\|x\|+\|y\|)$ for all $x, y \in X$.

If \|\|$: V \rightarrow \mathbb{R}$ fulfils conditions $1-3$, then we say that $(X,\|\|, K)$ is a quasi$\beta$-normed space.

A quasi- $\beta$-normed space is called a quasi- $\beta$ Banach space, if any Cauchy sequence converges.

Remark 8. 1. For any normed space $(X,\|\|)$ the function \|\|$_{p}:=\|\|^{p}$ defines a quasi- $\beta$-norm on $X$ with $\beta=p$ and $K=2^{p-1}$ for $p \geq 1$ (cf. Remark 3 ).

2. Any quasi- $\beta$-normed space $(X,\|\|, K)$ is also a dq- $K$-metric space by defining $d(x, y):=\|x-y\|$. Then $d$ is translation invariant, i.e., $d(x+$ $z, y+z)=d(x, y)$ for all $x, y, z$. Moreover, $d(\lambda x, \lambda y)=|\lambda|^{\beta} d(x, y)$ for all $x, y \in X$ and $\lambda \in \mathbb{K}$.

3. Let $(X, d)$ be a dq- $K$-metric space (with some $K \geq 1$ ). Then, given any elements $x_{1}, x_{2}, \ldots, x_{n+1} \in X$ with $n \in \mathbb{N}, n>1$, we have the inequality

$$
d\left(x_{1}, x_{n+1}\right) \leq \sum_{i=1}^{n-1} K^{i} d\left(x_{i}, x_{i+1}\right)+K^{n-1} d\left(x_{n}, x_{n+1}\right)
$$

and, as a consequence, the simpler to apply one

$$
d\left(x_{1}, x_{n+1}\right) \leq \sum_{i=1}^{n} K^{i} d\left(x_{i}, x_{i+1}\right)
$$

holds true. 
4. In a quasi- $\beta$-normed space $X$ the corresponding generalized triangle inequalities

$$
\begin{aligned}
\left\|\sum_{i=1}^{n} x_{i}\right\| & \leq \sum_{i=1}^{n-1} K^{i}\left\|x_{i}\right\|+K^{n-1}\left\|x_{n+1}\right\| \\
\left\|\sum_{i=1}^{n} x_{i}\right\| & \leq \sum_{i=1}^{n} K^{i}\left\|x_{i}\right\|
\end{aligned}
$$

hold true.

All those inequalities can be easily proved by induction.

The topic of Hyers-Ulam stability is a very important branch in the theory of functional equations. A survey and further references may be found for example in $[6,15]$.

One of the first and most important results now will axiomatically be termed as Hyers-Ulam property.

Definition 3. Let $(P,+)$ and $(G,+)$ be groupoids (i.e., $P$ and $G$ are a nonempty sets and $+: P^{2} \rightarrow P,+: G^{2} \rightarrow G$ inner operations). Let furthermore $\Phi_{1}, \Phi_{2}: P \rightarrow[0, \infty)$ and $\varphi_{1}, \varphi_{2}: P^{2} \rightarrow[0, \infty)$ be given functions and $d: G \times$ $G \rightarrow[0, \infty)$. Then we say that the pair $(P, G)$ has the Hyers-Ulam property (with respect to $\left(\varphi_{i}, \Phi_{i}\right)$ ), if for all functions $g: P \rightarrow G$ such that

$$
\begin{array}{ll}
d(g(u+v), g(u)+g(v)) \leq \varphi_{1}(u, v), & u, v \in P, \\
d(g(u)+g(v), g(u+v)) \leq \varphi_{2}(u, v), & u, v \in P,
\end{array}
$$

there is some function $a: P \rightarrow G$ such that

$$
\begin{array}{lc}
a(u+v)=a(u)+a(v), & u, v \in P, \\
d(a(u), g(u)) \leq \Phi_{1}(u), & u \in P, \\
d(g(u), a(u)) \leq \Phi_{2}(u), & u \in P .
\end{array}
$$

Remark 9. Let us recall that a groupoid $(P,+)$ is square symmetric provided

$$
2 x+2 y=2(x+y), \quad x, y \in P,
$$

where $2 x:=x+x$.

If a groupoid $(P,+)$ is square symmetric, then by induction we can prove that, for every $x, y \in P$,

$$
2^{n}(x+y)=2^{n} x+2^{n} y, \quad n \in \mathbb{N},
$$

where $2^{n+1} x=2\left(2^{n} x\right)$ for $n \in \mathbb{N}$.

We say that a groupoid $(P,+)$ is uniquely divisible by 2 if, for each $x \in P$, there is a unique $y \in P$ with $x=2 y$; we write $2^{-1} x:=y$. We define the notion $2^{-n} x$ for $x \in P$ by

$$
2^{-(n+1)} x=2^{-1}\left(2^{-n} x\right), \quad n \in \mathbb{N} .
$$


If a groupoid $(P,+)$ is uniquely divisible by 2 and square symmetric, then (again by induction), for every $x, y \in P$, we get

$$
2^{-n}(x+y)=2^{-n} x+2^{-n} y, \quad n \in \mathbb{N} .
$$

Clearly every abelian semigroup is square symmetric. Also, if $\mathbb{F}$ is a field, $A, B \in \mathbb{F}, X$ is a linear space over $\mathbb{F}, \gamma \in X$, and we define $\hat{+}: X^{2} \rightarrow X$ by

$$
x \widehat{+} y:=A x+B y+\gamma,
$$

then it is easy to check that $(X, \widehat{+})$ is a square symmetric groupoid (see, e.g., [16] for further information).

Note yet that, if $(H, \widehat{+})$ is a square symmetric groupoid, $D$ is a nonempty set, $h: D \rightarrow H$ is a bijection and we define a binary operation $*: D^{2} \rightarrow D$ by: $a * b:=h^{-1}(h(a) \hat{+} h(b))$ for $a, b \in D$, then $(D, *)$ is a square symmetric groupoid.

If $d$ is a dq- $K$-metric in a groupoid $(H,+)$, then we say that a function $g: H^{2} \rightarrow H$ is continuous (with respect to $d$ ) provided, for any sequences $\left(x_{n}\right)_{n=1}^{\infty}$ and $\left(y_{n}\right)_{n=1}^{\infty}$ in $H$ such that $x_{n} \rightarrow x$ and $y_{n} \rightarrow y$ with some $x, y \in H$, we have $g\left(x_{n}, y_{n}\right) \rightarrow g(x, y)$.

The following theorem is an auxiliary result. It is well-known in the metric spaces and usually attributed to PAŞC GĂVRUŢĂ [17]; however a result by Gian LUigi FORTI [14] is much more general, probably too general to have realized its consequences and special cases. We present a proof of it for dq- $K$ metric spaces (if $K=1$, then it corresponds to the results in [18] to certain extent).

Theorem 2. Let $(G,+)$ and $(X,+)$ be square symmetric groupoids, $X$ be uniquely divisible by 2 , and $d$ be a complete $d q-K$-metric in $X$ such that

$$
d\left(2^{-1} x, 2^{-1} y\right) \leq \xi d(x, y), \quad x, y \in X,
$$

with some $\xi>0$. Let the operation + in $X$ be continuous (with respect to $d$ ) and $\varphi_{i}: G \times G \rightarrow[0, \infty), i=1,2$, satisfy

$$
\begin{aligned}
& \Phi_{i}(x):=K^{2} \xi \sum_{j=0}^{\infty}(K \xi)^{j} \varphi_{i}\left(2^{j} x, 2^{j} x\right)<\infty, \quad x \in G, i=1,2, \\
& \lim _{j \rightarrow \infty} \xi^{j} \varphi_{i}\left(2^{j} x, 2^{j} y\right)=0, \quad x, y \in G, i=1,2 .
\end{aligned}
$$

Then the pair $(G, X)$ has the Hyers-Ulam property with respect to $\left(\varphi_{i}, \Phi_{i}\right)$. More exactly speaking, given $f: G \rightarrow X$ such that

$$
\begin{array}{ll}
d(f(x+y), f(x)+f(y)) \leq \varphi_{1}(x, y), & x, y \in G, \\
d(f(x)+f(y), f(x+y)) \leq \varphi_{2}(x, y), & x, y \in G,
\end{array}
$$


there is an additive function $\alpha: G \rightarrow X$, i.e., $\alpha(x+y)=\alpha(x)+\alpha(y)$ for all $x, y \in G$, such that

$$
d(\alpha(x), f(x)) \leq \Phi_{1}(x), \quad d(f(x), \alpha(x)) \leq \Phi_{2}(x), \quad x \in G .
$$

This is the only additive function $\alpha: G \rightarrow X$ which, given $L \geq 1$, satisfies

$$
d(\alpha(x), f(x)) \leq L \cdot \Phi_{1}(x), \quad d(f(x), \alpha(x)) \leq L \cdot \Phi_{2}(x), \quad x \in G .
$$

Moreover, (18) holds.

Proof. Given $n \in \mathbb{N}_{0}$ (nonnegative integers) and $f$ as in the theorem let $f_{n}: G \rightarrow X$ be defined by

$$
f_{n}(x):=2^{-n} f\left(2^{n} x\right) .
$$

Then $f_{0}=f$ and

$d\left(f_{n+1}(x), f_{n}(x)\right)=d\left(2^{-(n+1)} f\left(2 \cdot 2^{n} x\right), 2^{-(n+1)} 2 f\left(2^{n} x\right)\right) \leq \xi^{n+1} \varphi_{1}\left(2^{n} x, 2^{n} x\right)$, $d\left(f_{n}(x), f_{n+1}(x)\right)=d\left(2^{-(n+1)} 2 f\left(2^{n} x\right), 2^{-(n+1)} f\left(2 \cdot 2^{n} x\right)\right) \leq \xi^{n+1} \varphi_{2}\left(2^{n} x, 2^{n} x\right)$ by the properties of $d$ and by the property of $f$ for $x=y\left(=2^{n} x\right)$.

Given $n \in \mathbb{N}_{0}$ and $m \in \mathbb{N}$ we get from (4)

$$
\begin{aligned}
d\left(f_{n+m}(x), f_{n}(x)\right) & \leq \sum_{j=1}^{m} K^{j} d\left(f_{n+j}(x), f_{n+j-1}(x)\right) \\
& \leq \sum_{j=1}^{m} K^{j} \xi^{n+j} \varphi_{1}\left(2^{n+j-1} x, 2^{n+j-1} x\right) \\
& \leq \sum_{j=1}^{m} K^{n+j} \xi^{n+j} \varphi_{1}\left(2^{n+j-1} x, 2^{n+j-1} x\right) \\
& =K \xi \sum_{l=n}^{n+m-1}(K \xi)^{l} \varphi_{1}\left(2^{l} x, 2^{l} x\right) \\
& \leq K \xi \sum_{l=n}^{\infty}(K \xi)^{l} \varphi_{1}\left(2^{l} x, 2^{l} x\right) .
\end{aligned}
$$

Analogously we have

$$
d\left(f_{n}(x), f_{n+m}(x)\right) \leq K \xi \sum_{l=n}^{\infty}(K \xi)^{l} \varphi_{2}\left(2^{l} x, 2^{l} x\right) .
$$

This implies that the sequence $\left(f_{n}(x)\right)_{n \in \mathbb{N}_{0}}$ is a Cauchy sequence for all $x \in G$. Accordingly $\alpha: G \rightarrow X$,

$$
\alpha(x):=\lim _{n \rightarrow \infty} f_{n}(x), \quad x \in G,
$$


is well-defined. Using the above estimate for $n=0$ and $m \in \mathbb{N}$ we get

$$
\begin{aligned}
d(\alpha(x), f(x)) & \leq K\left(d\left(\alpha(x), f_{m}(x)\right)+d\left(f_{m}(x), f(x)\right)\right) \\
& \leq K\left(d\left(\alpha(x), f_{m}(x)\right)+K^{2} \xi \sum_{l=0}^{\infty}(K \xi)^{l} \varphi_{1}\left(2^{l} x, 2^{l} x\right) .\right.
\end{aligned}
$$

Now, taking the limit for $m \rightarrow \infty$ shows that

$$
d(\alpha(x), f(x)) \leq K^{2} \xi \sum_{l=0}^{\infty}(K \xi)^{l} \varphi_{1}\left(2^{l} x, 2^{l} x\right)=\Phi_{1}(x), \quad x \in G .
$$

Similarly we obtain

$$
d(f(x), \alpha(x)) \leq K^{2} \xi \sum_{l=0}^{\infty}(K \xi)^{l} \varphi_{2}\left(2^{l} x, 2^{l} x\right)=\Phi_{2}(x), \quad x \in G .
$$

We still must show that $\alpha$ is additive. Since $G$ and $X$ are square symmetric (cf. (9) and (10)),

$$
\begin{aligned}
d\left(f_{n}(x+y), f_{n}(x)+f_{n}(y)\right)= & d\left(2^{-n} f\left(2^{n} x+2^{n} y\right), 2^{-n}\left(f\left(2^{n} x\right)+f\left(2^{n} y\right)\right)\right) \\
\leq & \xi^{n} \varphi_{1}\left(2^{n} x, 2^{n} y\right), \\
d\left(f_{n}(x)+f_{n}(y), f_{n}(x+y)\right) \leq & \xi^{n} \varphi_{2}\left(2^{n} x, 2^{n} y\right), \\
d(\alpha(x+y), \alpha(x)+\alpha(y)) \leq & K d\left(\alpha(x+y), f_{n}(x+y)\right) \\
& +K^{2} d\left(f_{n}(x+y), f_{n}(x)+f_{n}(y)\right) \\
& +K^{2} d\left(f_{n}(x)+f_{n}(y), \alpha(x)+\alpha(y)\right), \\
d(\alpha(x)+\alpha(y), \alpha(x+y)) \leq & K d\left(\alpha(x)+\alpha(y), f_{n}(x)+f_{n}(y)\right) \\
& +K^{2} d\left(f_{n}(x)+f_{n}(y), f_{n}(x+y)\right) \\
& +K^{2} d\left(f_{n}(x+y), \alpha(x+y)\right)
\end{aligned}
$$

which for $n \rightarrow \infty$ implies $d(\alpha(x+y), \alpha(x)+\alpha(y))=0$ and $d(\alpha(x)+\alpha(y), \alpha(x+$ $y))=0$ (because (12) holds and + is continuous), whence $\alpha(x+y)=\alpha(x)+$ $\alpha(y)$.

If, finally $\gamma$ is additive with $d(\gamma(x), f(x)) \leq L \Phi_{1}(x)$ and $d(f(x), \gamma(x)) \leq$ $L \Phi_{2}(x)$ for all $x$, we get that

$$
\begin{aligned}
& d(\gamma(x), \alpha(x)) \leq K(d(\gamma(x), f(x))+d(f(x), \alpha(x))) \leq K\left(L \Phi_{1}(x)+\Phi_{2}(x)\right), \\
& d(\alpha(x), \gamma(x)) \leq K(d(\alpha(x), f(x))+d(f(x), \gamma(x)))+\leq K\left(\Phi_{1}(x)+L \Phi_{2}(x)\right) .
\end{aligned}
$$

Using this for $2^{n} x$ in place of $x$ and observing that $\gamma\left(2^{n} x\right)=2^{n} \gamma(x)$ and $\alpha\left(2^{n} x\right)=2^{n} \alpha(x)$ we obtain

$$
\begin{aligned}
d(\gamma(x), \alpha(x)) & =d\left(2^{-n} \gamma\left(2^{n} x\right), 2^{-n} \alpha\left(2^{n} x\right)\right) \leq \xi^{n} d\left(\gamma\left(2^{n} x\right), \alpha\left(2^{n} x\right)\right) \\
& \leq \xi^{n} K\left(L \Phi_{1}\left(2^{n} x\right)+\Phi_{2}\left(2^{n} x\right)\right), \\
d(\alpha(x), \gamma(x)) & \leq \xi^{n} K\left(\Phi_{1}\left(2^{n} x\right)+L \Phi_{2}\left(2^{n} x\right)\right)
\end{aligned}
$$


for $x \in X$ and $n \in \mathbb{N}$. Finally, in view of (11), we see that (with $n \geq 2$ )

$$
\begin{aligned}
\xi^{n} \Phi_{i}\left(2^{n} x\right) & :=K^{2} K^{-n} \xi \sum_{j=0}^{\infty}(K \xi)^{j+n} \varphi_{i}\left(2^{j+n} x, 2^{j+n} x\right) \\
& =K^{2-n} \xi \sum_{j=n}^{\infty}(K \xi)^{j} \varphi_{i}\left(2^{j} x, 2^{j} x\right) \\
& \leq \xi \sum_{j=n}^{\infty}(K \xi)^{j} \varphi_{i}\left(2^{j} x, 2^{j} x\right) \rightarrow 0 \text { for } n \rightarrow \infty
\end{aligned}
$$

for $x \in G$ and $i=1,2$. Thus, by (19) and (20), $d(\gamma(x), \alpha(x))=0$ and $d(\alpha(x), \gamma(x))=0$ for $x \in G$, whence $\gamma=\alpha$.

Remark 10. Let $p \geq 1$ and

$$
d(x, y)=(\max \{x-\lfloor y\rfloor, 0\})^{p}, \quad x, y \in \mathbb{R} .
$$

Then, according to Remarks 3 and $4, d$ is a dq- $2^{p-1}$-metric in $\mathbb{R}$. For such $d$ conditions (13) and (14) take the form

$$
\begin{array}{ll}
f(x+y)-\lfloor f(x)+f(y)\rfloor \leq \varphi_{1}(x, y)^{1 / p}, & x, y \in G, \\
f(x)+f(y)-\lfloor f(x+y)\rfloor \leq \varphi_{2}(x, y)^{1 / p}, & x, y \in G .
\end{array}
$$

This shows that using the notion of dq- $K$-metrics we can deal in a unified way with stability in some somewhat unusual situations.

Remark 11. If $d$ in Theorem 2 is continuous, then letting $m \rightarrow \infty$ in (16) and (17) (with $n=0$ ), we get the following estimations

$$
d(\alpha(x), f(x)) \leq \frac{1}{K} \Phi_{1}(x), \quad d(f(x), \alpha(x)) \leq \frac{1}{K} \Phi_{2}(x), \quad x \in G,
$$

which are a bit better than (15).

Note that, in general, a dq- $K$-metric does not need to be continuous with respect to itself.

A special case of a definition in [7] is the following.

Definition 4. Let $S$ be a non-empty set, $(P,+)$ and $(W,+)$ be groupoids, $\pi: S \rightarrow P$ be surjective and let $p: P \rightarrow S$ be a selection with respect to $\pi$, i.e., $\pi \circ p=\operatorname{id}_{P}$ or $p(u) \in \pi^{-1}(u)$ for all $u \in P$. Then $f: S \rightarrow W$ is called a solution of a (generalized) radical equation iff

$$
f(p(\pi(x)+\pi(y)))=f(x)+f(y), \quad x, y \in S .
$$

Corollary 2.2 in that paper implies the following result (under the assumptions as in Definition 4).

Theorem 3. Assume that $P$ has a neutral element and that $W$ is a monoid. Then $f: S \rightarrow W$ satisfies (23) if and only if there is some additive function $\alpha: P \rightarrow W$ such that $f=\alpha \circ \pi$. 
Remark 12. Under the assumptions of Definition 4, define an inner binary operation $\hat{+}$ in $S$ by:

$$
x \widehat{+} y:=p(\pi(x)+\pi(y)), \quad x, y \in S .
$$

Then equation (23) can be rewritten in the form

$$
f(x \widehat{+} y)=f(x)+f(y), \quad x, y \in S .
$$

Further, note that if the groupoid $(P,+)$ is square symmetric, then so is the groupoid $(S, \widehat{+})$ and thus we can use Theorem 2 to obtain some stability results concerning $(23)$, provided $(W,+)$ is square symmetric. An example of such a more concrete result is given in the next section (Theorem 4).

\section{Concrete results}

The first investigations on the stability of the radical functional equation was for the special case of (23), namely equation (40), for $f$ mapping $\mathbb{R}$ to some quasi- $\beta$ Banach space (see [21]). Replacing 2 by any positive integer $n$ in this equation we now formulate and prove the following theorem concerning stability of equation $(23)$ with $\pi: \mathbb{R} \rightarrow \pi(\mathbb{R})$ defined by $\pi(x)=x^{n}$ and $p: \pi(\mathbb{R}) \rightarrow \mathbb{R}$ by $p(u):=\sqrt[n]{u}$.

The next theorem is a simple consequence of Theorem 2 (see Remark 12).

Theorem 4. Let $(X,\|\cdot\|, K)$ be a quasi- $\beta$-Banach space, $n \in \mathbb{N}, A, B, C, a, b \in$ $\mathbb{R}, c \in X, \alpha:=a+b \neq 0$ and, in the case of even $n, A, B, C$ be nonnegative. Assume moreover that $\tau: \mathbb{R} \rightarrow \mathbb{R}$ is given by:

$$
\tau(x):=\sqrt[n]{(A+B) x^{n}+C}, \quad x \in \mathbb{R},
$$

and $\psi: \mathbb{R} \times \mathbb{R} \rightarrow[0, \infty[$ satisfies

$$
\begin{aligned}
& \Psi(x):=K \sum_{j=0}^{\infty}\left(\frac{K}{\alpha^{\beta}}\right)^{j+1} \psi\left(\tau^{j}(x), \tau^{j}(x)\right)<\infty, \\
& \lim _{j \rightarrow \infty} \frac{\psi\left(\tau^{j}(x), \tau^{j}(y)\right)}{\alpha^{\beta j}}=0, \quad x, y \in \mathbb{R} .
\end{aligned}
$$

Then, given $F: \mathbb{R} \rightarrow X$ such that

$$
\left\|F\left(\sqrt[n]{A x^{n}+B y^{n}+C}\right)-a F(x)-b F(y)-c\right\| \leq \psi(x, y), \quad x, y \in \mathbb{R},
$$

there is a unique solution $\gamma: \mathbb{R} \rightarrow X$ of the functional equation

$$
\gamma\left(\sqrt[n]{A x^{n}+B y^{n}+C}\right)=a \gamma(x)+b f \gamma(y)+c, \quad x, y \in \mathbb{R}
$$

such that $\|F(x)-\gamma(x)\| \leq \Psi(x)$ for all $x \in \mathbb{R}$. 
Proof. It is easy to check that the inner binary operations $+_{1}$ in $\mathbb{R}$ and $+_{2}$ in $X$,

$$
\begin{aligned}
x+{ }_{1} y & :=\sqrt[n]{A x^{n}+B y^{n}+C}, \quad x, y \in \mathbb{R}, \\
u+{ }_{2} w & :=a u+b w+c, \quad u, w \in X,
\end{aligned}
$$

are square symmetric (see Remarks 9 and 12). Moreover, the groupoid $\left(X,+_{2}\right)$ is uniquely divisible by 2 with

$$
2^{-1} u=\frac{1}{a+b}(u-c), \quad u \in X .
$$

Note yet that $X$ is a dq- $K$-metric space with a complete and invariant dq$K$-metric $d, d(x, y):=\|x-y\|$. So, we have a special case of Theorem 2 with $(G,+)=\left(\mathbb{R},+_{1}\right),(X,+)=\left(X,+_{2}\right), \varphi_{1}=\varphi_{2}=\psi$ and $\xi=(a+b)^{-\beta}$.

Remark 13. The result in [21] for $n=2$ (but only when $A=B=a=b=1$, $c=0, C=0$, and $F(0)=0$ ) is of similar flavour. It makes use of some transformation specific to that case.

Remark 14. [1] contains a result for the case $n=3$, which seems to be false. It claims that the condition $\left\|f\left(\sqrt[3]{x^{3}+y^{3}}\right)-f(x)-f(y)\right\| \leq \psi(x, y)$, under the assumptions $\Psi(x):=\sum_{j=1}^{\infty} 2^{-j \beta} \psi\left(2^{j / 3} x, 2^{j / 3} x\right)<\infty$ and $\lim _{n \rightarrow \infty} 2^{-n \beta}$ $\psi\left(2^{n / 3} x, 2^{n / 3} y\right)=0$, implies that $F$ defined by

$$
F(x):=\lim _{n \rightarrow \infty} 2^{-n} f\left(2^{n / 3} x\right)
$$

is a solution of the corresponding equation, which also satisfies $\|f(x)-F(x)\| \leq$ $K 2^{-\beta} \Psi(x)$ for all $x$.

This result can't be true in general. If, for example $\|x\|=|x|^{p}$ (with $X=\mathbb{R}$ and $p>1$ ), we define $f(x):=x^{3}+x$ and put $\psi(x, y):=\left|\sqrt[3]{x^{3}+y^{3}}-x-y\right|^{p}$. Then the hypotheses are satisfied. Moreover, in view of the formula for $F$, necessarily $F(x)=x^{3}$, thus $f(x)-F(x)=x$ and $\|f(x)-F(x)\|=|x|^{p}$.

Observe $\psi(x, x)=|2-\sqrt[3]{2}|^{p}|x|^{p}$ and $\beta=p$. Hence

$$
\begin{aligned}
\Psi(x) & =\frac{K}{2^{\beta}} \sum_{j=1}^{\infty} \frac{\psi\left(2^{j / 3} x, 2^{j / 3} x\right)}{2^{\beta j}}=\frac{K}{2^{p}}|2-\sqrt[3]{2}|^{p}|x|^{p} \sum_{j=1}^{\infty} \frac{\left(2^{j / 3}\right)^{p}}{2^{p j}} \\
& =K\left|1-2^{-2 / 3}\right|^{p} 2^{-2 p / 3} \frac{1}{1-2^{-2 p / 3}}|x|^{p} .
\end{aligned}
$$

The result cited would imply that $|x|^{p}=\|F(x)-f(x)\| \leq \Psi(x)$ which here would result in

$$
1 \leq K\left|1-2^{-2 / 3}\right|^{p} 2^{-2 p / 3} \frac{1}{1-2^{-2 p / 3}}
$$


Note that the $K$ corresponding to that quasi- $\beta$ norm has to be $\geq 2^{p-1}$ which for $p=2$ results in $K \geq 2$. But for $p=2$ and $K=2$

$$
K\left|1-2^{-2 / 3}\right|^{p} 2^{-2 p / 3} \frac{1}{1-2^{-2 p / 3}} \leq 0.19
$$

contradicting the fact that it should be $\geq 1$.

\section{Stability of the radical functional equation, abstract version}

The assumption that the groupoids (under consideration) are square symmetric is very important in Theorem 2 and consequently also in Theorem 4 . Below we provide a quite abstract theorem showing that, also without that assumption, some stability issues of the radical functional equation (23) can be reduced to the stability problems of the corresponding equation of homomorphism.

Let us start with the following definition.

Definition 5. Let $d$ be a dq-K-metric in a groupoid $(G,+)$. We say that $d$ is subinvariant, provided

$$
d(x, y) \leq d(x+z, y+z), \quad x, y, z \in G .
$$

Remark 15. Clearly, every invariant dq- $K$-metric is subinvariant. The converse is not true in general. For instance, let $G=[0, \infty), a, b$ be positive reals and $d$ be given by: $d(x, y)=a x^{2}+b y^{2}$ for $x, y \in G$. Then $d$ is a subinvariant dq-metric in $G$, which is not invariant.

In what follows we assume that $S$ is a nonempty set, $(P,+)$ and $(G,+)$ are groupoids, $d: G \times G \rightarrow[0, \infty)$ is a dq- $K$-metric on $G$, with a constant $K \geq 1$, $\pi: S \rightarrow P$ is surjective, and $p: P \rightarrow S$ is a selection with respect to $\pi$.

Now, we are in a position to present that abstract stability result concerning the radical functional equation (23).

Theorem 5. Let $\psi_{1}, \psi_{2}: S^{2} \rightarrow[0, \infty)$ and $\Phi_{1}, \Phi_{2}: P \rightarrow[0, \infty)$. Assume that $(P, G)$ has the Hyers-Ulam property with respect to $\varphi_{i}$ and $\Phi_{i}$, where

$$
\varphi_{i}(u, v):=\psi_{i}(p(u), p(v)), \quad u, v \in P .
$$

Suppose that one of the following two conditions is valid.

(i) $d$ is subinvariant.

(ii) Groupoid P has a neutral element 0 and

$$
\tau_{1}(y):=\sup _{z \in G} d(z+y, z)<\infty, \quad \tau_{2}(y):=\sup _{z \in G} d(z, z+y)<\infty, \quad y \in G .
$$

Then, for any $f: S \rightarrow G$ satisfying

$$
\begin{array}{ll}
d\left(f(p(\pi(x)+\pi(y)), f(x)+f(y)) \leq \psi_{1}(x, y),\right. & x, y \in S, \\
d\left(f(x)+f(y), f(p(\pi(x)+\pi(y))) \leq \psi_{2}(x, y),\right. & x, y \in S,
\end{array}
$$


there is a solution $F: S \rightarrow G$ of the radical equation (23) such that

$$
\begin{array}{ll}
d(F(x), f(x)) \leq K\left(\Phi_{1}(\pi(x))+\chi_{1}(x)\right), & x \in S, \\
d(f(x), F(x)) \leq K\left(\Phi_{2}(\pi(x))+\chi_{2}(x)\right), & x \in S,
\end{array}
$$

where, in the case of $(i)$,

$$
\begin{aligned}
& \chi_{1}(z)=\inf _{y_{0} \in S} K\left[\psi_{1}\left(p(\pi(z)), y_{0}\right)+\psi_{2}\left(z, y_{0}\right)\right], \\
& \chi_{2}(z)=\inf _{y_{0} \in S} K\left[\psi_{1}\left(z, y_{0}\right)+\psi_{2}\left(p(\pi(z)), y_{0}\right)\right],
\end{aligned}
$$

and, in the case of $(i i)$,

$$
\chi_{j}(z)=\inf _{x_{0} \in \pi^{-1}(\{0\})} K\left[\psi_{j}\left(z, x_{0}\right)+\tau_{j}\left(f\left(x_{0}\right)\right)\right], \quad j=1,2 .
$$

Proof. Let $f: S \rightarrow G$ satisfy (30) and (31) and define $g: P \rightarrow G$ by $g:=f \circ p$. Then

$$
\begin{array}{ll}
d(g(u+v), g(u)+g(v)) \leq \psi_{1}(p(u), p(v))=\varphi_{1}(u, v), & u, v \in P \\
d(g(u)+g(v), g(u+v)) \leq \psi_{2}(p(u), p(v))=\varphi_{2}(u, v), & u, v \in P .
\end{array}
$$

This may be seen in the following way: For $u, v \in P$ let $x:=p(u), y:=p(v)$. Clearly $\pi(x)=u, \pi(y)=v$, since $\pi \circ p=\mathrm{id}_{P}$. Thus

$$
\begin{aligned}
g(u+v) & =f(p(u+v))=f(p(\pi(p(u))+\pi(p(v)))=f(p(\pi(x)+\pi(y))), \\
g(u) & =f(p(u))=f(x), \quad g(v)=f(p(v))=f(y) .
\end{aligned}
$$

Then (30) with $p(x)=u, p(y)=v$ implies (34). Analogously we obtain (35).

Since, by assumption, $(P, G)$ has the Hyers-Ulam property with respect to $\varphi_{i}$ and $\Phi_{i}$, there is some additive function $\alpha: P \rightarrow G$ such that $d(\alpha(u), g(u)) \leq$ $\Phi_{1}(u)$ and $d(g(u), \alpha(u)) \leq \Phi_{2}(u)$ for all $u \in P$. In terms of $f$ this means that

$$
\begin{aligned}
& d(\alpha(\pi(x)), f(p(\pi(x)))) \leq \Phi_{1}(\pi(x)), \quad x \in S, \\
& d(f(p(\pi(x))), \alpha(\pi(x))) \leq \Phi_{2}(\pi(x)), \quad x \in S .
\end{aligned}
$$

Let $F:=\alpha \circ \pi$. Then, by (36) and (37),

$$
\begin{aligned}
d(F(x), f(x)) & =d(\alpha(\pi(x)), f(x)) \\
& \leq K[d(\alpha(\pi(x)), f(p(\pi(x))))+d(f(p(\pi(x))), f(x))] \\
& \leq K\left(\Phi_{1}(\pi(x))+\beta_{1}(x)\right), \quad x \in S, \\
d(f(x), F(x)) & =d(f(x), \alpha(\pi(x))) \\
& \leq K[d(f(x), f(p(\pi(x)))+d(f(p(\pi(x))), \alpha(\pi(x)))] \\
& \leq K\left(\Phi_{2}(\pi(x))+\beta_{2}(x)\right), \quad x \in S,
\end{aligned}
$$

where

$$
\beta_{1}(x)=d(f(p(\pi(x))), f(x)), \quad \beta_{2}(x)=d(f(x), f(p(\pi(x)))), \quad x \in S .
$$


Note that, in the case of (i), by (29), we have

$$
\begin{aligned}
d(f(z), f(w)) \leq & d\left(f(z)+f\left(y_{0}\right), f(w)+f\left(y_{0}\right)\right) \\
\leq & K\left[d\left(f(z)+f\left(y_{0}\right), f\left(p\left(\pi(z)+\pi\left(y_{0}\right)\right)\right)\right)\right. \\
& \left.+d\left(f\left(p\left(\pi(w)+\pi\left(y_{0}\right)\right)\right), f(w)+f\left(y_{0}\right)\right)\right] \\
\leq & K\left[\psi_{1}\left(z, y_{0}\right)+\psi_{2}\left(w, y_{0}\right)\right] .
\end{aligned}
$$

for every $z, w, y_{0} \in S$ with $\pi(z)=\pi(w)$. Clearly, this shows that

$$
\begin{aligned}
& \beta_{1}(z) \leq \inf _{y_{0} \in S} K\left[\psi_{1}\left(p(\pi(z)), y_{0}\right)+\psi_{2}\left(z, y_{0}\right)\right]=\chi_{1}(z), \\
& \beta_{2}(z) \leq \inf _{y_{0} \in S} K\left[\psi_{1}\left(z, y_{0}\right)+\psi_{2}\left(p(\pi(z)), y_{0}\right)\right]=\chi_{2}(z)
\end{aligned}
$$

for every $z \in S$. This and (38) and (39) yield (32) and (33).

Now, assume that $P$ has a neutral element 0 . Let $x_{0} \in S$ satisfy $\pi\left(x_{0}\right)=0$. Then

$$
\begin{aligned}
d\left(f(p(\pi(x))), f(x)+f\left(x_{0}\right)\right) & =d\left(f\left(p\left(\pi(x)+\pi\left(x_{0}\right)\right)\right), f(x)+f\left(x_{0}\right)\right) \\
& \leq \psi_{1}\left(x, x_{0}\right), \quad x \in S, \\
d\left(f(x)+f\left(x_{0}\right), f(p(\pi(x)))\right) & \leq \psi_{2}\left(x, x_{0}\right), \quad x \in S .
\end{aligned}
$$

Hence we obtain the following estimate for the distance between $f(x)$ and $f(p(\pi(x)))$ :

$$
\begin{aligned}
d(f(p(\pi(x))), f(x)) & \leq K\left[d\left(f\left(p(\pi(x)), f(x)+f\left(x_{0}\right)\right)+d\left(f(x)+f\left(x_{0}\right), f(x)\right)\right]\right. \\
& \leq K\left[\psi_{1}\left(x, x_{0}\right)+\tau_{1}\left(f\left(x_{0}\right)\right)\right], \quad x \in S, \\
d(f(x), f(p(\pi(x)))) & \leq K\left[d\left(f(x), f(x)+f\left(x_{0}\right)\right)+d\left(f(x)+f\left(x_{0}\right), f(p(\pi(x)))\right]\right. \\
& \leq K\left[\tau_{2}\left(f\left(x_{0}\right)\right)+\psi_{2}\left(x, x_{0}\right)\right], \quad x \in S .
\end{aligned}
$$

This and (38) and (39) imply (32) and (33) also when (ii) holds.

Remark 16. If $(i i)$ holds, $G$ has a neutral element 0 and $d$ is invariant, then $\tau_{1}(y)=d(y, 0)$ and $\tau_{2}(y)=d(0, y)$ for each $y \in G$ and consequently $\chi_{i}, i=1,2$, have the form

$$
\begin{aligned}
& \chi_{1}(z)=\inf _{x_{0} \in \pi^{-1}(\{0\})} K\left[d\left(f\left(x_{0}\right), 0\right)+\psi_{1}\left(z, x_{0}\right)\right], \\
& \chi_{2}(z)=\inf _{x_{0} \in \pi^{-1}(\{0\})} K\left[d\left(0, f\left(x_{0}\right)\right)+\varphi_{2}\left(z, x_{0}\right)\right]
\end{aligned}
$$

for $z \in S$, which are a bit different than those obtained in the case of $(i)$ and depend on $f$. In some of such situations we can derive from the assumptions an estimation of the values of $d\left(f\left(x_{0}\right), 0\right)$ and $d\left(0, f\left(x_{0}\right)\right)$, independent of the form of $f$. This is the case for instance for the equation

$$
f\left(\sqrt{x^{2}+y^{2}}\right)=f(x)+f(y)
$$


in the class of functions $f: \mathbb{R} \rightarrow \mathbb{R}$. Then conditions (30) and (31) take the forms

$$
\begin{aligned}
& d\left(f\left(\sqrt{x^{2}+y^{2}}\right), f(x)+f(y)\right) \leq \psi_{1}(x, y), \\
& d\left(f(x)+f(y), f\left(\sqrt{x^{2}+y^{2}}\right)\right) \leq \psi_{2}(x, y),
\end{aligned}
$$

which with $x=y=x_{0}=0$ yield

$$
\begin{aligned}
& d(0, f(0))=d(f(0), 2 f(0)) \leq \psi_{1}(0,0), \\
& d(f(0), 0)=d(2 f(0), f(0)) \leq \psi_{2}(0,0) .
\end{aligned}
$$

\section{Acknowledgements}

This work is funded by Jouf University, Kingdom of Saudi Arabia, under the research Project Number 39/600.

Open Access. This article is distributed under the terms of the Creative Commons Attribution 4.0 International License (http://creativecommons.org/licenses/by/4.0/), which permits unrestricted use, distribution, and reproduction in any medium, provided you give appropriate credit to the original author(s) and the source, provide a link to the Creative Commons license, and indicate if changes were made.

Publisher's Note Springer Nature remains neutral with regard to jurisdictional claims in published maps and institutional affiliations.

\section{References}

[1] Alizadeh, Z., Ghazanfari, A.G.: On the stability of a radical cubic functional equation in quasi- $\beta$-spaces. J. Fixed Point Theory Appl. 18(4), 843-853 (2016)

[2] Altun, I., Sola, F., Simsek, H.: Generalized contractions on partial metric spaces. Topol. Appl. 157(18), 2778-2785 (2010)

[3] Harandi, A.A.: Metric-like spaces, partial metric spaces and fixed points. Fixed Point Th. Appl. (2012), Article ID: 204

[4] Boriceanu, M., Bota, M., Petruşel, A.: Multivalued fractals in $b$-metric spaces. Cent. Eur. J. Math. 8(2), 367-377 (2010)

[5] Brzdęk, J.: Remarks on solutions to the functional equations of the radical type. Adv. Theory Nonlinear Anal. Appl. 1, 125-135 (2017)

[6] Brzdęk, J., Popa, D., Raşa, I., Xu, B.: Ulam Stability of Operators, Mathematical Analysis and Its Applications v. 1. Academic Press, Oxford (2018)

[7] Brzdęk, J., Schwaiger, J.: Remarks on solutions to a generalization of the radical functional equations. Aeq. Math. 92, 975-991 (2018)

[8] Bukatin, M., Kopperman, R., Matthews, S.G., Pajoohesh, S,H.: Partial metric spaces. Am. Math. Mon. 116, 708-718 (2009)

[9] Czerwik, S.: Contraction mappings in b-metric spaces. Acta Math. Univ. Ostrav. 1(1), 5-11 (1993)

[10] Czerwik, S.: Nonlinear set-valued contraction mappings in $b$-metric spaces. Atti Sem. Math. Fis. Univ. Modena 46, 263-276 (1998) 
[11] Deza, M.M., Deza, E.: Encyclopedia of Distances. Springer, Berlin (2009)

[12] Ding, Y., Xu, T.-Z.: Approximate solution of generalized inhomogeneous radical quadratic functional equations in 2-Banach spaces. J. Ineq. Appl. 31, 13 (2019)

[13] Dung, N.V., Hang, V.T.L.: The generalized hyperstability of general linear equations in quasi-Banach spaces. J. Math. Anal. Appl. 462, 131-147 (2018)

[14] Forti, G.L.: An existence and stability theorem for a class of functional equations. Stochastica 4(1), 23-30 (1980)

[15] Forti, G.L.: Hyers-Ulam stability of functional equations in several variables. Aeq. Math. 50(1-2), 143-190 (1995)

[16] Forti, G.L.: Continuous increasing weakly bisymmetric groupoids and quasi-groups in R. Math. Pannonica 8, 49-71 (1997)

[17] Găvruţa, P.: A generalization of the Hyers-Ulam-Rassias stability of approximately additive mappings. J. Math. Anal. Appl. 184(3), 431-436 (1994)

[18] Hejmej, B.: Stability of functional equations in dislocated quasi-metric spaces. Ann. Math. Sil. 32, 215-225 (2018)

[19] Karapinar, E., Erha, I.M.: Fixed point theorems for operators on partial metric spaces. Appl. Math. Lett. 24, 1894-1899 (2011)

[20] Khodaei, H., Eshaghi Gordji, M., Kim, S.S., Cho, Y.J.: Approximation of radical functional equations related to quadratic and quartic mappings. J. Math. Anal. Appl. 395(1), 284-297 (2012)

[21] Kim, S.S., Cho, Y.J., Eshaghi Gordji, M.: On the generalized Hyers-Ulam-Rassias stability problem of radical functional equations. J. Inequal. Appl. 2012, 13 (2012)

[22] Maligranda, L., Aoki, T.: (1910-1989). In: Proceedings of the International Symposium on Banach and Function Spaces II, Kitakyushu, Japan, 2006, Yokohama Publishers, pp. 1-23 (2008)

[23] Maligranda, L.: A result of Tosio Aoki about a generalization of Hyers-Ulam stability of additive functions - a question of priority. Aeq. Math. 75, 289-296 (2008)

[24] Paluszyński, M., Stempak, K.: On quasi-metric and metric spaces. Proc. Am. Math. Soc. 137(12), 4307-4312 (2009)

[25] Rahman, M.U., Sarwar, M.: Some new fixed point theorems in dislocated quasi-metric spaces. Palest. J. Math. 5, 171-176 (2015)

[26] Rassias, J.M., Kim, H.-M.: Generalized Hyers-Ulam stability for general additive functional equations in quasi- $\beta$-normed spaces. J. Math. Anal. Appl. 356(1), 302-309 (2009)

[27] Sarwar, M., Rahman, M.U., Ali, G.: Some fixed point results in dislocated quasi metric (dq-metric) spaces. J. Inequal. Appl. 2014, 278 (2014)

[28] Schroeder, V.: Quasi-metric and metric spaces. Conform. Geom. Dyn. 10, 355-360 (2006)

Janusz Brzdęk

Faculty of Applied Mathematics

AGH University of Science and Technology

Mickiewicza 30

30-059 Kraków

Poland

e-mail: brzdek@agh.edu.pl 
El-sayed El-hady

Mathematics Department, College of Science

Jouf University

P.O. Box 2014 Sakaka

Saudi Arabia

e-mail: elsayed_elhady@ci.suez.edu.eg

and

Basic Science Department, Faculty of Computers and Informatics Suez Canal University

Ismailia 41522

Egypt

Jens Schwaiger

Institute of Mathematics and Scientific Computing

University of Graz

Graz

Austria

e-mail: jens.schwaiger@uni-graz.at

Received: March 31, 2019

Revised: July 3, 2019 